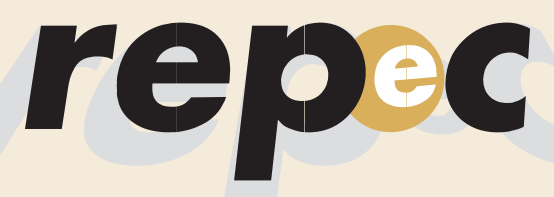

REPeC, Brasília, v. 9, n. 1, art. 5, p. 84-100, jan./mar. 2015 Disponível online em www.repec.org.br DOl: http://dx.doi.org/10.17524/repec.v9i1.1110

\title{
O Valor Adicionado dos Cursos de Ciências Contábeis: um Estudo na Região Sudeste do Brasil
}

\begin{abstract}
Resumo
Nos últimos anos, a profissão contábil tem passado por diversas mudanças. O papel do contador na sociedade tem evoluído, tornando a sua função mais sofisticada e sua formação acadêmica primordial para a execução das tarefas das empresas. Nesse contexto, este artigo busca identificar os fatores que explicam o Valor Adicionado pelas Instituições de Ensino Superior aos alunos do curso de Ciências Contábeis. Para tanto, utiliza-se os resultados da prova de conhecimentos específicos do Exame Nacional de Desempenho de Estudantes (Enade) 2009, tendo como amostra 251 cursos de Ciências Contábeis da região Sudeste. Na análise empírica dos dados, fez-se uso das estatísticas descritivas e de modelos de regressão linear. Com base na metodologia do valor adicionado, aplicou-se a teoria da Função de Produção, controlando-se características individuais e socioeconômicas dos alunos. Os resultados sugerem que os principais determinantes do Valor Adicionado são os seguintes: a existência de um plano de ensino, a quantidade de alunos que recebem bolsas de estudos e o tamanho do corpo discente.
\end{abstract}

Palavras-chave: Ciências Contábeis; Ensino Superior; Valor Adicionado.

\section{Silvana Cristina dos Santos Mestre em Ciências Contábeis pela Fucape Business School e Professor II da Faculdade de Ciências Gerenciais de Manhuaçu. Contato: Av. Getúlio Vargas, 733. Bairro: Coqueiro. Manhuaçu. MG. CEP.: 36900-000. \\ E-mail: silvanacssantos@hotmail.com}

\section{Cristiano Machado Costa}

Ph.D. em Economia pela University of Pennsylvania e Professor Assistente II na Universidade do Vale do Rio dos Sinos. Contato: Av. Unisinos, 950. Prédio 5A, Sala 304, Bairro: Cristo Rei. São Leopoldo. RS. CEP.: 93022-000.

E-mail: cristianocosta@unisinos.br 


\section{Introdução}

A profissão contábil vem evoluindo sob a influência de mudanças econômicas, sociais e tecnológicas (Domingues \& Schlindwein, 2007), dentre elas, destacam-se: o avanço tecnológico e informacional, a implantação do Sistema Público de Escrituração Digital, e as alterações na própria estrutura contábil, como a adoção das International Financial Reporting Standards (IFRS) (Miranda, Casa Nova \& Cornacchione Júnior, 2012). Nesse cenário, a profissão contábil tem ocupado posição de destaque, resultando em crescimento de oportunidades para o profissional da área (Parisoto, Grande \& Fernandes, 2006; Byrne \& Flood, 2008). Como consequência, é requerido desse profissional um perfil adequado à grandeza das mudanças do mercado atual, que tem se mostrado cada vez mais dinâmico e competitivo (Domingues \& Schlindwein, 2007; Parisoto, Grande \& Fernandes, 2006).

Essas mudanças afetam de formas diversas o ensino da Contabilidade no Brasil (Miranda, Casa Nova \& Cornacchione Júnior, 2012). Como responsáveis pela formação dos profissionais da área, as Instituições de Ensino Superior (IES) desempenham o papel de ser o instrumento condutor da Ciência Contábil a atender às novas expectativas da sociedade (Parisoto, Grande \& Fernandes, 2006). Sabe-se que o ensino superior tem se destacado por uma grande expansão em diversas áreas do conhecimento (Souza \& Machado, 2011). Neste ambiente, o curso de Ciências Contábeis vem apresentando um expressivo aumento da quantidade de cursos e de vagas tanto em IES públicas quanto nas privadas (Araújo, Camargos \& Camargos, 2011).

De acordo com Araújo, Camargos e Camargos (2011, p.1), um problema que se acentuou com a expansão rápida do ensino superior foi "a qualidade do ensino e o resultado final do processo de aprendizagem, o desempenho discente, que surge como uma questão crucial na pauta de discussão da educação brasileira." A necessidade crescente e iminente de se conhecer a qualidade do ensino que tem sido ofertado e que tipo de profissional tem sido formado é preocupação também de outros países (Zhang, 2009; Yunker, 2005; Saavedra \& Saavedra, 2011; Byrne \& Flood, 2008).

Nesse cenário, órgãos governamentais implantam diferentes metodologias de avaliação do ensino superior (Araújo, Camargos \& Camargos, 2011). Uma delas, que vem sendo implantada e utilizada em vários países, é a do valor adicionado (Value-Added). O termo Valor Adicionado origina-se da economia e, na educação, passou a ser compreendido, em termos gerais, como a contribuição da escola, ao aluno (Fincher, 1985). Assim, esse método busca medir a contribuição pura da escola e o efeito de suas políticas e práticas para o desempenho do aluno (Doran \& Izum, 2004). Ou seja, a contribuição livre de todas as outras fontes de realização de um aluno (Meyer, 1997).

No Brasil, Soares, Ribeiro e Castro (2001) estimaram o Valor Adicionado de IES em Minas Gerais para os cursos de Direito, Administração e Engenharia Civil. Os autores possuíam dados internos da Universidade Federal de Minas Gerais (UFMG) sobre desempenho prévio dos alunos no vestibular. Porém, os dados eram apenas para os que fizeram o vestibular da UFMG e foi este o dado usado como base para cálculo do valor agregado. Apesar desta limitação, o estudo apontou que o nível socioeconômico influenciava o aprendizado dos alunos e serviu como uma primeira discussão sobre a importância da metodologia de Valor Adicionado na avaliação do ensino superior no Brasil. Já Santos (2012) analisou o efeito de características individuais e institucionais sobre o desempenho de estudantes de Ciências Contábeis que participaram do ENC-Provão de 2002 e 2003 e do Exame Nacional de Desempenho dos Estudantes (Enade), de 2006. Entretanto, sua análise limitou-se ao uso de microdados, além de utilizar somente dados de alunos concluintes. Outros trabalhos na área de Ciências Contábeis, relacionados com o desempenho, têm se concentrado principalmente em analisar possíveis variáveis que influenciam o resultado (nota) dos alunos concluintes no Enade (Cruz \& Teixeira, 2012; Souza, Machado \& Machado, 2011; Araújo, Camargos \& Camargos, 2011).

O tema Valor Adicionado na educação pode ser visto amplamente como objeto de pesquisas e estudos em outros países. No Brasil, especialmente no ensino superior, foram verificados poucos trabalhos relacionados a essa temática. Assim, o presente artigo buscou preencher essa lacuna, além de estender 
as contribuições anteriores relacionadas aos determinantes do Valor Adicionado pelas IES nos cursos de Ciências Contábeis. A pergunta de pesquisa deste estudo pode ser colocada da seguinte forma: Quais os fatores que explicam o Valor Adicionado pelas IES aos alunos do curso de Ciências Contábeis em relação aos conhecimentos específicos avaliados no Enade na região Sudeste do Brasil?

O perfil de estudantes ingressantes em uma IES é heterogêneo, e as diferenças de desempenho são consequências também de outros fatores, entre outros, desempenho prévio características individuais e familiares (Clark, 1983; Lareau, 1987). Por considerar esses fatores, a metodologia do Valor Adicionado tem sido considerada apropriada e promissora para se mensurar o efeito puro de uma instituição de ensino (Hanushek \& Taylor, 1990; Meyer 1997; Doran \& Izumi, 2004; McDonnell, Chellman, Littman \& Crook, 2013), já utilizada por vários países, e vem sendo tema de vários estudos. Nesse âmbito, justifica-se a importância e relevância deste trabalho, haja vista ainda ser voltado para educação e estar inserido no contexto da avaliação da qualidade do sistema de ensino, especificamente, no ensino de nível superior, em que a prestação de contas é cada vez mais necessária e requerida por vários interessados (Miranda, Casa Nova \& Cornacchione Júnior, 2012, Domingues \& Schlindwein, 2007).

\section{Referencial Teórico e Empírico}

\subsection{Avaliação da Educação Superior no Brasil}

Em 2004, foi implantando o Sistema Nacional de Avaliação da Educação Superior, que é operacionalizado pelo Instituto Nacional de Estudos e Pesquisas Educacionais Anísio Teixeira (Inep) e é formado por três componentes: avaliação das instituições, dos cursos e do desempenho dos estudantes (Inep, 2013a).

A avaliação dos estudantes é realizada por intermédio do Enade, que é componente curricular obrigatório dos cursos de graduação, aplicado com periodicidade máxima trienal. E que “[...] aferirá o desempenho dos estudantes em relação aos conteúdos programáticos previstos nas diretrizes curriculares do respectivo curso de graduação" (Lei no 10.861, 2004).

Compõem essa prova questões referentes à formação geral e específica. Dez questões fazem parte da formação geral, com peso de $25 \%$ e comum aos estudantes de todos os cursos selecionados; e trinta questões que fazem parte da formação específica, com peso de $75 \%$ e diferenciada para cada um dos cursos de graduação. Ambas as provas contêm questões discursivas e de múltipla escolha nas duas partes (Inep, 2013b). Até o ano de 2010 participavam da prova dois grupos de estudantes, os quais se encontravam em momentos diferentes de sua graduação e eram submetidos à mesma prova: um grupo, considerado ingressante, cursando o final do primeiro ano e outro grupo tido como concluinte, que se encontra no final do último ano do curso (Inep, 2013b). A partir de 2011, o Inep tomou a decisão de dispensar os estudantes ingressantes de realizar a prova do Enade e, em substituição, utilizar o resultado do Exame Nacional do Ensino Médio (Enem). Desse modo, a partir do ano de 2011, somente estudantes concluintes realizaram a prova do Enade (Inep, 2011).

\subsection{Método do Valor Adicionado}

Valor adicionado segundo Goldstein e Thomas (1996) é o quantitativo acrescentado por uma instituição de ensino, por via de suas políticas, práticas e processos internos, ao desempenho acadêmico dos alunos. Sob a perspectiva de linha de tempo, "muitos pesquisadores consideram o conceito de Valor Adicionado na educação como a ferramenta analítica mais importante que surgiu em ciências pedagógicas nos últimos 20 anos" [Tradução nossa] (Malach \& Malcik, 2010, p.125). Em um contexto de preocupação com a qualidade do ensino superior, no qual indicadores de resultados educacionais passaram a ser cada vez mais utilizados para avaliar a eficácia da educação (Meyer, 1997), o método do Valor Adicionado ga- 
nhou ainda mais força como objeto de investigação, e passou a ser considerado um indicador apropriado para medir e atribuir maior responsabilidade no que se refere ao ganho educacional (Meyer, 1997; Meyer \& Dokumaci, 2009).

Os modelos de Valor Adicionado, conhecidos internacionalmente pelo termo VAM, são um conjunto de procedimentos estatísticos que utilizam dados longitudinais. Em especial, todos os fatores possíveis que contribuem para o progresso no desempenho do aluno (Meyer, 1997; Doran \& Izumi, 2004). A intenção principal é medir a contribuição da escola e/ou professores para o desempenho do estudante, isolada de todas as outras fontes de realização do estudante (Meyer \& Dokumaci, 2009).

Várias abordagens podem ser encontradas para VAM (Raudenbush, 2004), e a escolha do modelo leva em consideração vários fatores, principalmente o tamanho da amostra a ser analisada, o conjunto de variáveis contextuais disponíveis e a frequência com que os alunos foram avaliados (Meyer, 1997; Doran \& Izumi, , 2004; Meyer \& Domakuci, 2009).

Nesse contexto, uma abordagem comum que tem sido utilizada para estimar o Valor Adicionado trata da Teoria da Função de Produção, em que a educação pode ser tratada como um processo de produção em que o nível educacional (desempenho) de um indivíduo é determinado pelas suas características pessoais, antecedentes familiares e insumos escolares (Hanushek, 1986; Hanushek \& Taylor, 1990). Uma vantagem dessa abordagem, segundo Taylor e Nguyen (2006), é permitir que a influência de fatores relacionados com o aluno e com a escola, no Valor Adicionado, sejam estimados separadamente.

Vários países já adotaram esse método de avaliação de desempenho educacional, tanto em nível de ensino superior como em outros níveis. Experiências práticas foram relatadas na Inglaterra, Polônia, República Checa, Filipinas, Austrália, Taiwan e em destaque nos Estados Unidos, onde é crescente o número de estudos relacionados (Taylor \& Nguyen, 2006; Jakubowski, 2008; Bacolod \& Tobias, 2006; Keeves, Hungi \& Afrassa, 2005; Kong \& Fu, 2012; Yunker, 2005; Malach \& Malcik, 2010).

Sendo assim, formas de aplicação prática pela adoção desse método são relatadas: Escolas e instituições de ensino têm adotado políticas de incentivos financeiros, pagamentos de bônus e aplicações de sanções para professores e funcionários, de acordo com o ganho adicionado ao aluno (Ladd \& Walsh, 2002; Jakubowski, 2008, Malach \& Malcik, 2010). E ainda para prestações de contas, para avaliarem seu desempenho, monitorando seu próprio progresso e fazendo comparações com seus concorrentes locais (Cunha \& Miller, 2014; Malach \& Malcik, 2010). Pais e alunos fazem uso das informações de Valor Adicionado na busca de uma escola apropriada (Meyer, 1997; Cunha \& Miller, 2014; Jakubowski, 2008). Governos, administradores e formuladores de políticas públicas têm sido grandes adeptos e usuários dessa ferramenta (Taylor \& Nguyen, 2006, Meyer \& Dokumaci, 2009). Em forma de feedback, as informações são utilizadas para apresentação ao público e transparência do investimento público no ensino (Zhang, 2009). Além de servirem de base para intervenção no processo de ensino para melhorar a eficiência institucional, com financiamento público, fixação de metas e recompensas e aplicações de penalidades (Cunha \& Miller, 2014). Contudo, críticas, limitações e desafios são apontados para o método apresentado. Meyer (1997) menciona que estimar o efeito das escolas sobre os resultados educacionais não é uma tarefa fácil, devido a deficiências metodológicas e falta de dados adequados. Ladd \& Walsh (2002) citam que medir o Valor Adicionado é um esforço compensativo, porém traz muitos desafios. Dessa forma, é preciso cuidado no desenvolvimento, na interpretação e utilização de medidas de Valor Adicionado da eficácia da escola (Ladd \& Walsh, 2002; Yunker, 2005; Bacolod \& Tobias, 2006). Everson, Feinauer e Sudweeks (2013) sugerem a não utilização de medidas de valor adicionado para a avaliação e classificação de professores, diante das dúvidas que ainda existem sobre o método. Papay (2012) afirma que modelos de Valor Adicionado que incluem apenas pontuações dos alunos em testes para medir desempenho podem estar sujeitos a muitas limitações. Amrein-Beardsley \& Collins (2012) consideram que, na avaliação e responsabilização de professores, as decisões de alto risco não devem ser feitas, baseando-se apenas nos indicadores de Valor Adicionado. 


\subsection{Estudos Anteriores}

Taylor \& Nguyen (2006) apresentam como principais conclusões de sua análise na Inglaterra: a proporção entre professores que trabalham meio expediente ou tempo integral é positivamente associada com aumento do Valor Adicionado. Já em escolas com maior quantidade de alunos de famílias pobres e maiores taxas de ausência autorizada, encontram-se pontuações mais baixas do Valor Adicionado.

Procurando avaliar o desempenho escolar nas Filipinas, Bacolod \& Tobias (2006) apontam que a maioria da variação no desempenho decorre de características em nível individual, sendo a educação dos pais e recursos familiares as variáveis que apresentaram maiores coeficientes positivo. A variável "instalações básicas" mostrou-se mais importante do que o tamanho da classe ou programas de formação de professores.

Examinando a utilidade potencial do conceito de Valor Adicionado em Contabilidade no ensino superior, Yunker (2005) utilizou como fonte de dados principais os resultados do exame para Certified Public Accountant (CPA), que habilita um contador público nos EUA. Os resultados da amostra pesquisada sugeriram a utilidade duvidosa de medidas de Valor Adicionado para fins de avaliação de desempenho das instituições de ensino superior em Contabilidade. Malach e Malcík (2010) abordam sobre a fase de ensaios-piloto e análises teóricas na República Checa para inclusão de medidas de Valor Adicionado no sistema nacional da educação. A conclusão obtida é de que é possível realizar nacionalmente estimativas de Valor Adicionado na educação.

Saavedra \& Saavedra (2011) estimaram o Valor Adicionado de uma educação universitária na Colômbia e sugeriram que o ensino superior aumenta novas competências essenciais para o estudante. Os maiores ganhos estão relacionados ao fato de o aluno estudar em uma universidade privada, ser do sexo feminino, ser de um fundo socioeconômico mais alto, ter uma mãe mais educada, estudar em tempo integral e que ter obtido maiores notas em seus testes de entrada na faculdade. Concluiu-se ainda que a pontuação de entrada dos estudantes na faculdade é o mais forte preditor de seu resultado final e as métricas, como percentual de professores credenciados com doutorado, percentual de professores trabalhando em tempo integral, seletividade (vagas/candidatos) e gastos por aluno, não foram correlacionadas com maiores ganhos em pensamento crítico, resolução de problemas, habilidades interpessoais e de comunicação.

O estudo de Jakubowski (2008) discute a implementação de estimativas de valor adicionado para escolas no ensino secundário da Polônia. O autor conclui que, apesar de alguns problemas metodológicos, modelos de Valor Adicionado são confiáveis e podem trazer benefícios para vários destinatários. Ainda pôde-se verificar que o aumento de despesas por estudante diminui a qualidade do ensino e escolas não públicas adicionaram mais valor ao estudante.

\subsubsection{Estudos Brasileiros na Área de Ciências Contábeis}

Souza \& Machado (2011) apontaram como determinantes do desempenho dos cursos de Ciências Contábeis no Enade 2006 o nível de conhecimento do aluno anterior ao seu ingresso em uma IES e em ordem decrescente; a escolaridade do pai; o esforço pessoal no curso; e a renda familiar.

Baseando-se no Enade 2009, Cruz \& Teixeira (2012) concluíram que não é possível afirmar que há relação entre os conteúdos curriculares classificados como formação básica, profissional e teórico-prática com as notas dos alunos no exame; e ainda a qualidade da organização pedagógica, cursos com maior número de docentes mestres e o tipo de instituição (pública ou privada) podem ter potencial relação com o desempenho dos alunos. Os principais achados do estudo de Santos (2012) foram: aspectos pessoais, como etnia, gênero e idade dos estudantes foram relacionados ao seu desempenho acadêmico; assim como positivamente, os coeficientes estimados de renda familiar e o efeito de o estudante ter cursado o ensino médio na escola pública; relação negativa significativa entre o desempenho e o fato de o estudante ser solteiro; e IES com maior proporção de docentes com titulação de mestrado ou de doutorado e com jornada integral, de 40 horas, ou com dedicação exclusiva ao ensino, produziram impactos positivos no desempenho acadêmico dos estudantes. 


\section{Metodologia}

Para se atingirem os objetivos propostos, foram utilizados os resultados do Enade 2009. Os dados foram coletados por meio dos relatórios disponíveis no portal do Inep. Conforme Inep (2009), participaram da avaliação em 2009 o total de 902 cursos de Ciências Contábeis. Inicialmente pretendia-se aplicar o estudo nos 902 cursos. Contudo, ao iniciar o processo de coleta dos dados, verificou-se que estavam organizados de forma desagregada. E esse processo por si só, exigiu meses de trabalho, tornando inviável pesquisar o universo total. Assim, foi selecionado como amostra o total de cursos avaliados na região Sudeste, que concentra o maior número de IES na área contábil (E-MEC, 2013), sendo a de maior representação no Enade 2009, com 357 cursos, concentrando 39,6\% do total nacional.

Dados os objetivos da pesquisa, tornou-se necessário excluir da amostra 106 instituições que não possuíam os dados necessários para a composição da base de dados. Logo, a amostra ficou constituída de 251 cursos (estão incluídos 14.467 alunos ingressantes e 11.796 concluintes), sendo 19 do Estado do Espírito Santo, 65 de Minas Gerais, 38 do Rio de Janeiro e 129 de São Paulo.

Para tratamento dos dados, foi realizada uma análise econométrica, por meio de regressão múltipla. Foi utilizada a abordagem da Teoria da Função de Produção, proposta por pesquisadores da área em estudo (Hanushek, 1986; Hanushek \& Taylor, 1990; Taylor \& Nguyen, 2006; Meyer, 1997). As variáveis do modelo foram estabelecidas, apoiando-se na literatura apresentada, conforme detalhado na Figura 1:

\begin{tabular}{|c|c|}
\hline Variáveis dependentes & Descrição \\
\hline ME_CE_CONC & Nota média dos alunos concluintes na área de componentes específicos. \\
\hline VALORADIC & $\begin{array}{l}\text { Estimativa do Valor Adicionado pelas instituições aos alunos, medido em pontos, } \\
\text { com relação ao valor médio (centrado em zero). }\end{array}$ \\
\hline \multicolumn{2}{|l|}{ Variáveis independentes } \\
\hline \multicolumn{2}{|l|}{ Relacionadas com o Aluno } \\
\hline ME_CE_ING & Nota média dos alunos ingressantes na área de componentes específicos. \\
\hline SOLT_CONC & Percentual de alunos concluintes solteiros. \\
\hline BRAN_CONC & Percentual de alunos concluintes brancos. \\
\hline NEG_CONC & Percentual de alunos concluintes negros. \\
\hline ATREP_CONC & Percentual de alunos concluintes em cuja casa mora acima de três pessoas. \\
\hline AC10SAL_CONC & $\begin{array}{l}\text { Percentual de alunos concluintes que possuem renda familiar acima de } 10 \\
\text { salários mínimos. }\end{array}$ \\
\hline TRABM40_CONC & Percentual de alunos concluintes que trabalham 40 horas semanais ou mais. \\
\hline \multicolumn{2}{|l|}{ Relacionadas com a Instituição } \\
\hline PROP_DOCD & Proporção de docentes no mínimo doutores. \\
\hline PROP_DOCM & Proporção de docentes no mínimo mestres. \\
\hline PROP_REG_PARC & Proporção de docentes que trabalham regime parcial ou integral. \\
\hline PROP_EQUIP_SUF & $\begin{array}{l}\text { Proporção de alunos que avaliaram positivamente os equipamentos } \\
\text { disponibilizados pela instituição. }\end{array}$ \\
\hline PROP_PLAN_ENSI & Proporção de alunos que avaliaram positivamente os planos de ensino. \\
\hline SIM_CONC & $\begin{array}{l}\text { Percentual de alunos concluintes que possuiu algum tipo de bolsa de estudos } \\
\text { para custear o curso. }\end{array}$ \\
\hline NUM_CONC & Número de alunos participantes como concluintes. \\
\hline N_DOCENT & Número de docentes de cada instituição de ensino. \\
\hline
\end{tabular}

Figura 1. sumário das variáveis utilizadas na análise dos dados. 
A partir da abordagem adotada e das variáveis estabelecidas, os modelos empíricos a serem estimados por mínimos quadrados foram assim definidos:

Primeiro Estágio:

ME_CE_CONC ${ }_{\mathrm{i}}=\beta_{0}+\beta_{1} \mathrm{ME}_{-} \mathrm{CE} \_I N G_{\mathrm{i}}+\beta_{2} \mathrm{SOLT} \_\mathrm{CONC}+\beta_{3} \mathrm{BRAN}_{-} \mathrm{CONC} \mathrm{i}_{\mathrm{i}}+\beta_{4} \mathrm{NEG}_{-} \mathrm{CONC} \mathrm{i}_{\mathrm{i}}$ $+\beta_{5}$ ATREP_CONC $+\beta_{6}$ AC10SAL_CONC $+\beta_{7}$ TRABM40_CONC ${ }_{i}+\varepsilon_{\mathrm{i}}$

Segundo Estágio:

VALORADIC $_{\mathrm{i}}=\gamma_{0}+\gamma_{1}$ PROP_DOCD $+\gamma_{2}$ PROP_DOCM ${ }_{\mathrm{i}}+\gamma_{3}$ PROP_REG_PARC $+\gamma_{4}$ PROP $(\text { EQUIP_SUF })_{i}+\gamma_{5}$ PROP_(PLAN_ENSI $)_{i}+\gamma_{6}$ SIM_CON $_{i}+\gamma 7 \mathrm{NUM}_{-} C O \mathrm{CNC}_{\mathrm{i}}+\gamma_{8} \mathrm{~N}_{-} \mathrm{DOCENT}_{\mathrm{i}}+\mathrm{u}_{\mathrm{i}}$

Em um primeiro estágio, estima-se o nível educacional (desempenho) de um indivíduo determinado pelo desempenho dos alunos ingressantes no Enade 2009 em conhecimentos específicos e as características pessoais e familiares dos alunos concluintes. E utiliza-se o resíduo como uma medida de Valor Adicionado (VALORADIC). Ou seja, todo o desempenho do aluno concluinte que não pode ser explicado por suas características individuais e desempenho dos ingressantes pode ser considerado o Valor Adicionado pela instituição. No segundo estágio, estima-se o efeito das características da instituição sobre o Valor Adicionado, o que permite verificar quanto do Valor Adicionado se deve às características da escola.

Deve-se ressaltar que o termo de erro estocástico da primeira regressão é a medida de Valor Adicionado estimado, levando-se em conta somente as características dos alunos. Esse método calcula o resíduo a partir de um modelo de regressão linear, controlando apenas fatores fora da influência da faculdade (McDonnell et al., 2013, Bailey \& Xu, 2012). Isso por que as instituições podem, por exemplo, tomar decisões sobre alocação de recursos que irão refletir a eficiência no que está se tentando medir. Assim, se esses recursos são incluídos, poderia haver riscos de resultados tendenciosos serem obtidos, já que o objetivo é medir o quão bem as instituições utilizam seus recursos (Kelchen \& Harris, 2012). À vista disso e apoiando-se no entendimento desses autores citados, os fatores sob o controle da instituição foram propositadamente excluídos na estimação do Valor Adicionado (primeiro estágio).

Destaca-se ainda que, quanto à variável independente do modelo que mede o resultado prévio de um determinado período de escolaridade (ME_CE_ING), a indicação apontada pela maioria dos autores é a de que os alunos que irão compor a estimativa do Valor Adicionado (com suas pontuações nos testes) sejam os mesmos que foram testados previamente. Entretanto o Enade, até o ano de 2010, não era aplicado a todos os alunos. O processo se dava por amostragem e não havia nenhum critério que incluísse na seleção os mesmos alunos avaliados como ingressantes e que, após três anos, eram avaliados como concluintes. Posto isso, uma alternativa para que tal situação não prejudicasse os resultados dessa pesquisa está fundamentada na prática da estimativa. Utilizou-se a nota dos alunos ingressantes nos conhecimentos específicos do Enade 2009, como Proxy, para o desempenho dos alunos concluintes no momento de ingresso. 


\subsection{Estatísticas Descritivas}

Apresenta-se a seguir a estatística descritiva das variáveis utilizadas. O número de ingressantes, ou seja, aqueles que tiveram suas notas incluídas no modelo como uma medida de desempenho em fase inicial do curso, foram em média 57 alunos. Caindo para a média de 46 alunos, estão os concluintes, aqueles que se valeram de suas notas para completar os dados como medida de desempenho final, próximo ao fim de sua graduação. Desse grupo, em média, 65\% são alunos solteiros; $68 \%$ são brancos; e 6,1\% são negros. Em termos de composição dos dados socioeconômicos, 30,2\% dos alunos concluintes moram com mais de três pessoas em casa, e ainda uma média de $12,9 \%$ e $78 \%$, respectivamente, têm renda familiar acima de 10 salários mínimos e trabalham 40 horas semanais ou mais.

Quanto aos insumos das instituições que participam da amostra, têm-se: (I) número médio de 28 docentes, entre os quais 10,8\% são doutores; (II) a proporção de docentes no mínimo mestres representou $51,2 \%$ médio do total; (III) a proporção de docentes que trabalham em regime parcial ou integral em média é de 44,8\%; (IV) 41,4\% dos alunos concluintes possuíram bolsa de estudos para custear o curso; (V) 73,5\% dos alunos avaliaram, positivamente, os equipamentos disponibilizados pela instituição; (VI) 49,9\% dos alunos avaliaram, positivamente, os planos de ensino dos professores.

Apresenta-se na Tabela 1 o sumário estatístico descritivo das notas dos alunos ingressantes e concluintes na avaliação do Enade 2009, em relação aos conhecimentos específicos da área de Contabilidade:

Tabela 1

Sumário estatístico descritivo das notas dos alunos no Enade 2009

\begin{tabular}{|c|c|c|c|c|c|c|}
\hline & Variáveis & Observações & Média & Mínimo & Máximo & Desvio Padrão \\
\hline \multirow{2}{*}{ Região Sudeste } & ME_CE_ING & 251 & 23,819 & 11,948 & 40,787 & 3,629 \\
\hline & ME_CE_CONC & 251 & 33,111 & 20,493 & 60,491 & 5,801 \\
\hline \multirow{2}{*}{ Espírito Santo } & ME_CE_ING & 19 & 24,167 & 18,067 & 28,575 & 2,811 \\
\hline & ME_CE_CONC & 19 & 32,965 & 24,425 & 46,900 & 6,721 \\
\hline \multirow{2}{*}{ Minas Gerais } & ME_CE_ING & 65 & 24,544 & 16,829 & 40,787 & 4,297 \\
\hline & ME_CE_CONC & 65 & 33,454 & 23,272 & 48,452 & 5,346 \\
\hline \multirow{2}{*}{ Rio de Janeiro } & ME_CE_ING & 38 & 24,364 & 16,950 & 33,861 & 4,347 \\
\hline & ME_CE_CONC & 38 & 34,371 & 22,133 & 60,491 & 7,153 \\
\hline \multirow{2}{*}{ São Paulo } & ME_CE_ING & 129 & 23,242 & 11,948 & 31,939 & 3,035 \\
\hline & ME_CE_CONC & 129 & 32,588 & 20,493 & 51,150 & 5,430 \\
\hline
\end{tabular}

Fonte: elaborado pelos autores a partir dos dados do Enade 2009.

Como pode ser observado na Tabela 1, na região Sudeste os alunos concluintes obtiveram desempenho superior, com uma média de 33,111 pontos, em relação aos alunos ingressantes, com uma média de 23,819 pontos. Os desvios-padrão indicaram que a variabilidade entre os concluintes foi superior $(5,801)$ do que entre os ingressantes $(3,629)$. Percebe-se que, em todos os estados, os alunos em fase final de curso tiveram notas médias maiores do que na fase inicial do curso, o que permite inferir que os alunos do curso de Ciências Contábeis da região Sudeste avaliados no Enade 2009, durante o período de sua formação, melhoraram seu desempenho no que tange aos conhecimentos específicos da área, em média de 9,292 pontos.

Deve-se destacar que a diferença de nota média entre os ingressantes e concluintes para a região Sudeste como um todo, bem como para cada um dos estados estudados, é, estatisticamente, diferente a um nível de significância de $1 \%$. Os valores t- calculados foram -21,51, -5,26, -10,47 e -7,37, para Sudeste, ES, MG, RJ e SP, respectivamente. Procurou-se compreender alguns dos fatores que determinam o aumento verificado no desempenho dos alunos, por meio da regressão múltipla, e esses resultados, assim como os da estimativa do valor adicionado, são apresentados na seção seguinte. 


\section{Análise e Discussão dos Resultados}

\subsection{Determinantes do Desempenho dos Alunos}

Os sumários estatísticos da regressão linear do primeiro estágio são apresentados nas Tabelas 2 a 4 .

Tabela 2

Sumário estatístico da regressão linear

\begin{tabular}{lcccccc}
\hline ME_CE_CONC & Coeficiente & Desvio-Padrão & $\mathbf{t}$ & $\mathbf{P}>|\mathbf{t}|$ & [95\% Intervalo de Confiança] \\
\hline ME_CE_ING & 0,690 & 0,094 & 7,36 & 0,000 & 0,506 & 0,875 \\
Constante & 16,669 & 2,175 & 7,67 & 0,000 & 12,387 & 20,952 \\
\hline Número de Observações = 251 & & & & & & \\
$\mathrm{R}^{2}=0,1865$ & & & & & \\
Estatística F $(1,249)=54,24(0,0000)$ &
\end{tabular}

Fonte: dados da pesquisa.

Nota-se na Tabela 2 que a relação positiva significativa é verificada entre a nota dos alunos ingressantes e as notas dos alunos concluintes, uma vez que o p-valor apresentou-se menor que 0,05 . Como neste trabalho o desempenho dos alunos ingressantes foi utilizado como proxy para o desempenho dos alunos concluintes no momento de ingresso, os resultados indicam que os conhecimentos prévios já trazidos pelo aluno no ingresso em uma instituição contribuem de forma significativa em seu desempenho. Em conformidade com esse resultado, têm-se os trabalhos anteriores de Wright, Fox, Murray, Carruthers \& Thrall (2012), Saavedra \& Saavedra (2011), Souza \& Machado (2011), além de Eskew \& Faley (1988) e Byrne \& Flood (2008), que são estudos aplicados também em cursos de Contabilidade. A inclusão desta variável no modelo resulta em um $\mathrm{R}^{2}$ de 0,186 . Ou seja, as variações da nota média do aluno quando ingressa conseguem explicar cerca de $18,6 \%$ da variabilidade das notas médias dos concluintes ao término do curso.

Na Tabela 3 demonstram-se os resultados de algumas variáveis de controle (características individuais do aluno, familiares e socioeconômicas) que foram incluídas no modelo de regressão:

Tabela 3

Sumário estatístico da regressão linear

\begin{tabular}{lcccccc}
\hline ME_CE_CONC & Coeficiente & Desvio-Padrão & $\mathbf{t}$ & $\mathbf{P}>|\mathbf{t}|$ & [95\% Intervalo de Confiança] \\
\hline ME_CE_ING & 0,617 & 0,092 & 6,73 & 0,000 & 0,437 & 0,798 \\
SOLT_CONC & 4,674 & 2,188 & 2,14 & 0,034 & 0,363 & 8,984 \\
BRAN_CONC & $-4,276$ & 2,519 & $-1,70$ & 0,091 & $-9,238$ & 0,687 \\
NEG_CONC & $-16,047$ & 5,770 & $-2,78$ & 0,006 & $-27,413$ & $-4,682$ \\
ATREP_CONC & $-5,250$ & 2,362 & $-2,22$ & 0,027 & $-9,903$ & $-0,597$ \\
Constante & 20,846 & 2,841 & 7,34 & 0,000 & 15,250 & 26,442 \\
\hline Número de Observações = 251 & & & & & & \\
R $=0,2410$ & & & & &
\end{tabular}

Fonte: dados da pesquisa. 
Verifica-se que os coeficientes que se mostraram significativos para um nível de significância de $5 \%$, foram: a) SOLT_CONC ( $\mathrm{t}=2,14$; $\mathrm{p}$-valor=0,034), indicando que um aumento de 10 pontos percentuais na proporção de alunos concluintes solteiros eleva o desempenho em média em 0,4674 pontos. Esse achado se desvia dos resultados de Santos (2012), que encontrou nessa relação um efeito significativo negativo; b) NEG_CONC ( $\mathrm{t}=-2,78$; $\mathrm{p}$-valor=0,006) - para esta variável observa-se relação negativa com o desempenho do aluno, estimando-se que um aumento de 10 pontos percentuais na proporção de alunos concluintes negros reduz em média 0,16047 pontos no desempenho de um aluno negro. Esse resultado é convergente com o trabalho de Wright et al. (2012) e divergente de Zhang (2009) e de Dale \& Krueger (2002), que, em seus estudos, não verificaram realização prejudicada para alunos negros; $c)$ ATREP_CONC $(t=-2,22 ; p-$ -valor $=0,027)$ - a relação negativa neste caso indica que o fato de o aluno morar com mais de três pessoas em casa piora o seu desempenho.

Em uma nova especificação, incluíram-se ainda duas novas variáveis de controle relacionadas com o aluno: a renda familiar e a situação do trabalho, cujos resultados são apresentados na Tabela 4.

Ratificando os resultados de Taylor \& Nguyen (2006), Bacolod \& Tobias (2006), Zhang (2009), Wright et al. (2012), Saavedra \& Saavedra (2011), a variável "renda familiar" (AC10SAL_CONC) mostrou-se positivamente relacionada ao desempenho e significante a 5\%. Cabe destacar que essa comparação independe do nível de renda das amostras, uma vez que se refere ao sinal e significância do parâmetro estimado. Estima-se que um aluno que tenha renda familiar acima de 10 salários mínimos possui um melhor desempenho, em média de 13,646 pontos (este efeito marginal, ao contrário, pode variar entre amostras). Já o fato de o aluno trabalhar em tempo integral (40 horas semanais ou mais) não demonstrou relação significativa ( $\mathrm{p}$-valor $=0,115)$ com as notas obtidas pelo aluno, para os dados desta pesquisa.

Tabela 4

\section{Sumário estatístico da regressão linear}

\begin{tabular}{|c|c|c|c|c|c|c|}
\hline ME_CE_CONC & Coeficiente & Desvio-Padrão & t & $P>|t|$ & \multicolumn{2}{|c|}{ [95\% Intervalo de Confiança] } \\
\hline ME_CE_ING & 0,450 & 0,092 & 4,90 & 0,000 & 0,269 & 0,631 \\
\hline SOLT_CONC & 5,592 & 2,164 & 2,58 & 0,010 & 1,329 & 9,855 \\
\hline BRAN_CONC & $-5,306$ & 2,655 & $-2,00$ & 0,047 & $-10,535$ & $-0,077$ \\
\hline NEG_CONC & $-14,931$ & 5,690 & $-2,62$ & 0,009 & $-26,139$ & $-3,722$ \\
\hline ATREP_CONC & $-9,288$ & 2,631 & $-3,53$ & 0,000 & $-14,470$ & $-4,106$ \\
\hline AC10SAL_CONC & 13,646 & 2,990 & 4,56 & 0,000 & 7,757 & 19,536 \\
\hline TRABM40_CONC & $-4,994$ & 3,158 & $-1,58$ & 0,115 & $-11,214$ & 1,226 \\
\hline Constante & 28,211 & 3,996 & 7,06 & 0,000 & 20,339 & 36,083 \\
\hline \multicolumn{7}{|c|}{ Número de Observações = 251} \\
\hline \multicolumn{7}{|l|}{$R^{2}=0,3396$} \\
\hline \multicolumn{7}{|c|}{ Estatística F $(7,243)=13,42(0,0000)$} \\
\hline
\end{tabular}

Fonte: dados da pesquisa.

Ressalta-se que a adição de novas variáveis no modelo não acarretou alterações relevantes nos coeficientes das outras variáveis, apenas a variável BRAN_CONC, que passou a ser significativa a 5\%, indicando uma possível relação negativa entre percentual de alunos brancos e seu desempenho. Isso que sugere a necessidade de uma investigação mais detalhada do motivo para obtenção desses sinais, especialmente, porque se apresentam contrários às expectativas advindas da literatura e, ainda, pela importância atual de questões relacionadas à raça, como os sistemas de cotas e políticas de reservas de vagas. Em suma, nota-se que a inclusão das variáveis "características individuais" e "familiares" resultou em um $\mathrm{R}^{2}$ de 0,33 , sugerindo que o modelo explica, aproximadamente, um terço da variabilidade das notas médias dos concluintes. 


\subsection{Valor Adicionado e seus Determinantes}

Apresenta-se na Tabela 5 o sumário estatístico descritivo do Valor Adicionado estimado no primeiro estágio:

Tabela 5

Sumário estatístico descritivo do Valor Adicionado estimado

\begin{tabular}{cccccc}
\hline Variáveis & Observações & Média & Mínimo & Máximo & Desvio Padrão \\
\hline VALORADIC & 251 & $-9,17 e-09$ & $-9,523$ & 16,513 & 4,714 \\
\hline
\end{tabular}

Fonte: dados da pesquisa.

Nota-se que como a variável VALORADIC é o resultado de um resíduo da regressão do primeiro estágio, ela tem uma média aproximada de 0 por definição. Observa-se que os valores se encontram entre -9,523 e máximo de 16,513, e essa distribuição apresenta um desvio-padrão de 4,714.

Na Figura 1, as instituições foram ordenadas de acordo com seu Valor Adicionado:

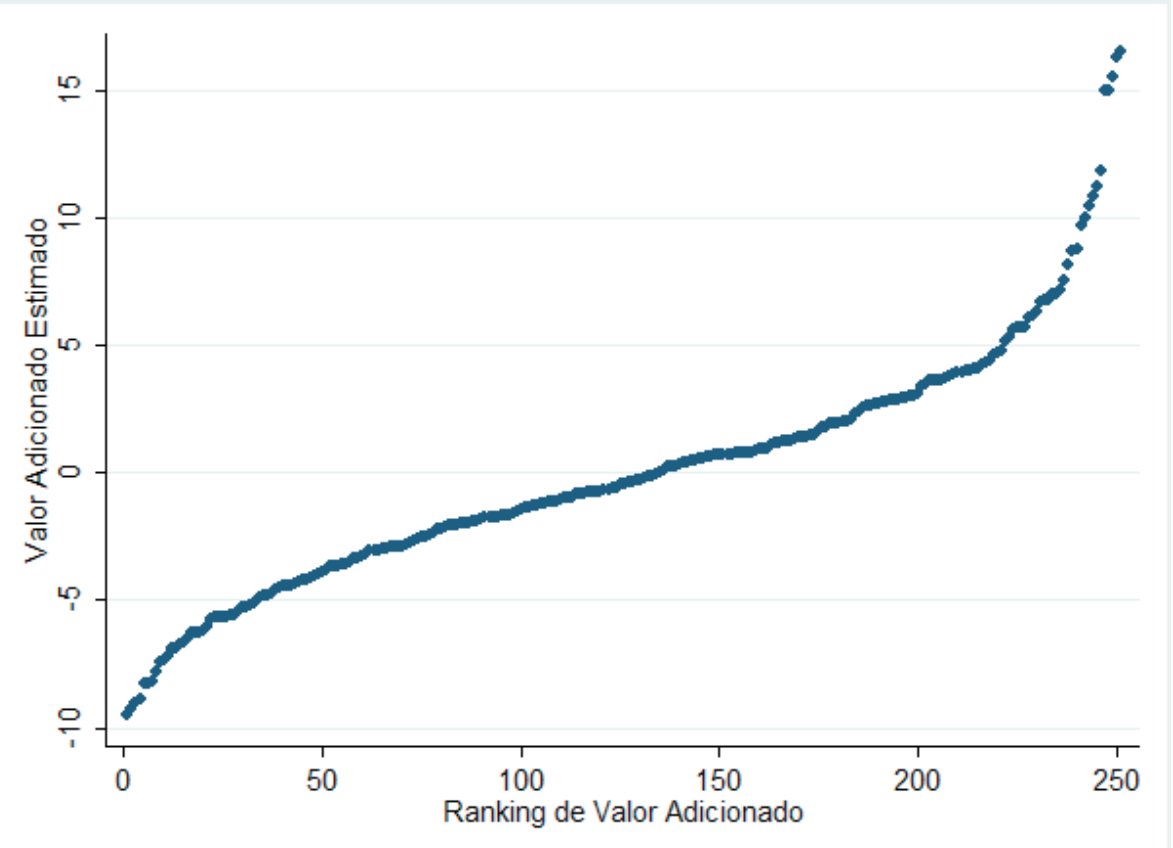

Figura 1. Ranking de Valor Adicionado estimado das instituições.

Fonte: dados da pesquisa.

A Figura 1 apresenta uma curva ascendente, demonstrando que as instituições que adicionaram menor valor estão posicionadas no lado esquerdo e as que adicionaram maior valor estão no lado direito. Existe também um conjunto de instituições que se destacam relativamente às outras, que adicionaram valor acima de 10 pontos. Para se conhecerem alguns dos possíveis determinantes deste Valor Adicionado, foi feita a regressão do Valor Adicionado estimado nas características da instituição de ensino (segundo estágio de estimação) e os resultados são apresentados nas Tabelas 6 e 7: 
Tabela 6

Sumário estatístico da regressão linear

\begin{tabular}{lcccccc}
\hline VALORADIC & Coeficiente & Desvio- Padrão & $\mathbf{t}$ & $\mathbf{P}>|\mathbf{t}|$ & [95\% Intervalo de Confiança] \\
\hline PROP_DOCD & $-4,669$ & 2,554 & $-1,83$ & 0,069 & $-9,699$ & 0,360 \\
PROP_DOCM & 2,968 & 1,548 & 1,92 & 0,056 & $-0,082$ & 6,018 \\
PROP_REG_PARC & 3,141 & 1,126 & 2,79 & 0,006 & 0,924 & 5,358 \\
Constante & $-2,423$ & 0,731 & $-3,31$ & 0,001 & $-3,863$ & $-0,982$ \\
\hline
\end{tabular}

Número de Observações $=251$

$\mathrm{R}^{2}=0,0497$

Estatística $\mathrm{F}(3,247)=5,17(0,0018)$

Fonte: dados da pesquisa.

Pode-se notar que não foram significativos ao nível de significância de 5\% os resultados para inferências quanto à relação entre o Valor Adicionado e a proporção de docentes no mínimo doutores (p-valor $=0,069)$ e a proporção de docentes no mínimo mestres ( $\mathrm{p}$-valor=0,056). Esses resultados vão de encontro aos de Saavedra \& Saavedra (2011) e divergem dos de Zhang (2009). Demonstra-se na Tabela 7 o resultado de outra especificação em que foram incluídas novas variáveis relacionadas com as instituições de ensino.

Observa-se na Tabela 7 que, quando incluídas as novas variáveis, a variável prop_reg_parc, que corresponde à proporção média de docentes que trabalham regime parcial ou integral, deixou de ser significativa ao nível de significância de 5\%, divergindo do estudo de Taylor \& Nguyen (2006). Com relação ao plano de ensino dos docentes, o coeficiente se mostrou positivamente significativo, inclusive a $1 \%$ de significância ( $\mathrm{p}$-valor=0,004), indicando que um bom plano de ensino (foi avaliado dessa forma pelo aluno) contribui de forma significativa no incremento do Valor Adicionado.

De igual modo, verificou-se relação positiva significativa entre as proporções médias de alunos que possuíram bolsa de estudos para custear o curso e o valor adicionado, uma vez que o p-valor apresentou-se em $0,029(<0,05)$.

Dessa forma, estima-se que o valor adicionado de uma instituição é acrescentado em média de 0,3028 pontos, quando a proporção de alunos com bolsa é elevada em 10 pontos percentuais. Uma possível causa para tal resultado é que, em muitos casos, os programas de bolsas de estudos (tanto do Governo por meio do Programa Universidade para Todos- ProUni, como das próprias IES) possuem exigências especiais para os estudantes, como o aproveitamento especial nas disciplinas cursadas. E isso demandará que o estudante se dedique mais aos estudos e naturalmente obtenha melhores resultados, o que impactará o Valor Adicionado da instituição. 
Tabela 7

Sumário estatístico da regressão linear

\begin{tabular}{lcccccc}
\hline VALORADIC & Coeficiente & Desvio- Padrão & $\mathbf{t}$ & $\mathbf{P}>\mathbf{| t} \mid$ & \multicolumn{1}{c}{ [95\% Intervalo de Confiança] } \\
\hline PROP_DOCD & $-2,957$ & 2,745 & $-1,08$ & 0,282 & $-8,363$ & 2,449 \\
PROP_DOCM & 2,067 & 1,624 & 1,27 & 0,204 & $-1,132$ & 5,266 \\
PROP_REG_PARC & 1,794 & 1,097 & 1,64 & 0,103 & $-0,367$ & 3,954 \\
PROP_EQUIP_SUF & 1,644 & 2,435 & 0,68 & 0,500 & $-3,153$ & 6,441 \\
PROP_PLAN_ENSI & 6,368 & 2,160 & 2,95 & 0,004 & 2,113 & 10,623 \\
SIM_CONC & 3,028 & 1,378 & 2,20 & 0,029 & 0,313 & 5,743 \\
NUM_CONC & $-0,016$ & 0,007 & $-2,10$ & 0,036 & $-0,030$ & $-0,001$ \\
N_DOCENT & 0,014 & 0,015 & 0,88 & 0,378 & $-0,017$ & 0,044 \\
Constante & $-6,826$ & 1,261 & $-5,41$ & 0,000 & $-9,309$ & $-4,343$ \\
\hline
\end{tabular}

Número de Observações $=251$

$\mathrm{R}^{2}=0,1878$

Estatística $\mathrm{F}(8,242)=6,70(0,0000)$

Fonte: dados da pesquisa.

Outra variável que se mostrou significativa a um nível de 5\% foi o número de alunos (NUM_CONC). Entretanto, verifica-se uma relação negativa, mostrando que, para o acréscimo de cada 1, aluno o Valor Agregado diminuiu em 0,016. Esse resultado se alinha com Saavedra \& Saavedra (2011) e com Taylor \& Nguyen (2006) e diverge de Zhang (2009), que sugeriu que tamanho da classe parece não ter afetado a qualidade da faculdade. Cabe destacar que o tamanho do corpo docente medido pela variável N_DOCENT não é significativo.

Nota-se ainda que a variável que representa a infraestrutura (PROP_EQUIP_SUF), sendo a proporção de alunos que consideraram que os equipamentos são suficientes, não se mostrou significativa ao nível de significância de 5\%. Em outros trabalhos, como de Bacolod \& Tobias (2006), os recursos da escola se mostraram significantes para explicar a variação do desempenho da escola. É o que se espera tanto dos recursos da escola como da qualidade do corpo docente: que produzam efeitos positivos no desempenho das instituições. Portanto, seria interessante outras investigações do mesmo grupo de instituições deste trabalho, para verificar, após serem controlados por outros fatores, se os recursos da infraestrutura e qualidade do corpo docente da instituição permaneceriam não interferindo em seus resultados.

Por fim, nota-se para esse modelo um $\mathrm{R}^{2}$ de 0,187 , indicando que esse conjunto de características da instituição explica, aproximadamente, $18,7 \%$ da variabilidade do Valor Adicionado da instituição de ensino (medido em notas) aos alunos. Assim, existem outros fatores que influenciam o Valor Adicionado das instituições e que não foram capturados pela regressão, e novos estudos poderão identificá-los.

\section{Considerações Finais}

O presente trabalho investigou os fatores que explicam o Valor Adicionado pelas instituições de ensino superior aos alunos do curso de Ciências Contábeis em relação aos conhecimentos específicos da área. Para tanto, empregou-se a metodologia do Valor Adicionado, que vem sendo adotada em vários países e permite conhecer o efeito exclusivo das instituições sobre o desempenho do estudante. Esse efeito é conhecido como Valor Adicionado porque exclui outros determinantes do desempenho, como, por exemplo, características particulares e socioeconômicas dos estudantes. Assim sendo, é possível obter uma estimativa de como a instituição de ensino está, efetivamente, contribuindo para o progresso do estudante.

Dessa forma, pode-se concluir que, tomando por base os dados do Enade 2009, as IES da região Sudeste adicionaram ganhos específicos da área para o aluno do curso de Ciências Contábeis. Os princi- 
pais determinantes do Valor Adicionado foram: plano de ensino, quantidade de alunos que utilizam bolsa de estudos e tamanho do corpo discente. Diante desses resultados, cabe destacar que, enquanto variáveis relacionadas com o corpo docente e infraestrutura pareceram não influenciar o desempenho da instituição, ressalta-se a relevância e importância da organização didático-pedagógica, que, conforme encontrado neste trabalho, pode motivar ganhos nos resultados da instituição de ensino.

Como conclusões adicionais, verificou-se, por um lado, que as variáveis explicativas "desempenho prévio do aluno", "o aluno ser negro", "o aluno ser branco", "o aluno ser solteiro", "morar com mais de três pessoas em casa" e "renda familiar" mostraram-se significantes para explicar o desempenho. Por outro lado, 'trabalhar em tempo integral (40 horas semanais ou mais) aparentou não se relacionar com o desempenho do estudante.

Várias prescrições educacionais podem emergir desse conjunto de resultados, podendo contribuir principalmente para reflexões e ações por parte das instituições de ensino, concernente à implicação de políticas e práticas adotadas que podem maximizar sua eficácia (independentemente de alterações ao longo do tempo nas características dos alunos que servem), com o propósito de melhorar o desempenho acadêmico do estudante, como, por exemplo: investimento em recursos didático-pedagógicos, maximização de programas de concessão de bolsas de estudos e elaboração de planos para controle da quantidade de alunos admitidos.

Além das instituições, esses resultados podem ser úteis para o Inep, responsável pela avaliação das IES no Brasil. Na elaboração deste estudo, verificou-se que o Indicador de Diferença entre os Desempenhos Observado e Esperado (IDD), que é uma das medidas de qualidade adotadas pelo Inep, apresenta semelhanças com os indicadores de Valor Adicionado, em seus objetivos, metodologia de cálculo, etc. Logo, toda a literatura apresentada e os resultados deste trabalho podem ser úteis para, sobretudo, despertar reflexões sobre a metodologia de cálculo utilizada atualmente para a obtenção do IDD. A finalidade é de contribuir para a produção de medidas de avaliação da qualidade do ensino mais justas e adequadas, que venham atender melhor a finalidade para a qual são empregadas. já que o IDD, além de ser um importante indicador de qualidade, ainda compõe o cálculo de outros indicadores de qualidade, como Conceito Preliminar de Curso (CPC) - esse posteriormente irá compor o Índice Geral de Cursos (IGC). Todos esses índices são publicados para o acesso do público em geral e, entre outras finalidades, são frequentemente utilizados pelas IES para divulgação de seu desempenho.

Outros estudos poderão investigar fatores determinantes do Valor Adicionado nos cursos de Ciências Contábeis em outros estados, bem como ampliar a amostra em nível nacional. Assim será possível verificar a existência de diferenças entre estados quanto às contribuições efetivas das IES para os alunos. Novas investigações poderão também pesquisar outros determinantes do desempenho dos alunos de Ciências Contábeis e compará-los, por exemplo, entre estados; e ainda pesquisar esses determinantes do desempenho por meio do resultado da prova do Exame de Suficiência em Contabilidade.

Outra sugestão seria aplicar a abordagem do Valor Adicionado, utilizando como medida de desempenho final as pontuações obtidas pelos estudantes no Exame de Suficiência em Contabilidade. Ainda poderia ser feita uma análise das IES em relação ao Valor Adicionado para o estudante, e o desempenho que esses estudantes obterão no Exame de Suficiência em Contabilidade, buscando saber se esse Valor Adicionado pelas instituições irá beneficiar o estudante no exame em questão. Por fim, também pode ser pertinente, em estudos futuros, medir o Valor Adicionado pelas IES, utilizando como proxy de desempenho final o valor do salário do estudante, quando estiver inserido no mercado de trabalho, após se formar.

Como limitações da presente pesquisa, devemos mencionar a utilização de uma amostra constituída pelo método de amostragem intencional, por meio de seleção, o que impossibilita generalizações dos resultados alcançados. Quanto às variáveis do modelo de Regressão, temos três limitações impostas pela base de dados. As notas médias dos estudantes de cada instituição obtidas em um teste como o Enade são apenas medidas aproximadas do conhecimento real do aluno. Ou seja, são uma proxy do real conhecimento acumulado do aluno. Além disto, usamos a nota dos alunos ingressantes obtidas no Enade 2009 como proxy para o desempenho dos alunos concluintes no momento de ingresso. Por fim, como as características individuais e socioeconômicas dos estudantes são declaradas (obtidas por meio de um questionários respondidos pelos alunos), existe a possibilidade de as questões respondidas pelos estudantes não representarem as suas reais condições. 


\section{Referências}

Amrein-Beardsley, A., \& Collins, C. (2012). The SAS Education Value-Added Assessment System (SAS EVAAS) in the Houston Independent School District (HISD): Intended and Unintended Consequences. Education Policy Analysis Archives, 20(12), pp.1-31.

Araújo, E. A. T., Camargos, M. A., \& Camargos, M. S. C. (2011). Desempenho Acadêmico de Discentes do Curso de Ciências Contábeis: Uma Análise dos Seus Fatores Determinantes em uma IES Privada. Anais do Encontro Nacional de Pós-graduação e Pesquisa em Administração, Rio de Janeiro, RJ, Brasil, 35.

Bacolod, M. P. \& Tobias, J. L. (2006). Schools, school quality and achievement growth: Evidence from the Philippines. Economics of Education Review, 25(6), pp. 619-632. doi: 10.1016/j.econedurev.2005.08.004

Bailey, T. \& XU, D. (2012) Input-adjusted graduation rates and college accountability: What is known from twenty years of research? Context for Success Working Paper.

Byrne, M. \& Flood, B. (2008). Examining the relationships among background variables and academic performance of first year accounting students at an Irish University. Journal of Accounting Education, 26(4), pp. 202-212. doi: 10.1016/j.jaccedu.2009.02.001

Clark, R. M. (1983) Family Life and School Achievement: Why Poor Black Children Succeed or Fail .Chicago: The University of Chicago Press.

Cruz, A. J. \& Teixeira, A. M. C. (2012) Desempenho dos Alunos no Enade de 2009: um estudo empírico a partir do conteúdo curricular dos cursos de Ciências Contábeis no Brasil. Anais do Encontro Nacional de Pós-graduação e Pesquisa em Administração, Rio de Janeiro, RJ, Brasil, 35.

Cunha, J. M. \& Miller, T. (2014) Measuring value-added in higher education: Possibilities and limitations in the use of administrative data. Economics of Education Review, 42, 64-77. doi:10.1016/j.econedurev.2014.06.001

Dale, S. B. \& Krueger, A. B. (2002). Estimating the payoff to attending a more selective college: An application of selection on observables and unobservables. Quarterly Journal of Economics, 117(4), pp. 1491-1527. doi: 10.1162/003355302320935089

Domingues, M. J. C. S. \& Schlindwein, A. C. (2013). O Ensino de Ciências Contábeis nas Instituições de Ensino Superior (IES) da Mesorregião do Vale do Itajaí - SC: uma análise das contribuições curriculares da Resolução Cne/Ces n. 10/2004. Anais do Encontro de Ensino e Pesquisa em Administração e Contabilidade, Recife, PE, Brasil, 1.

Doran, H.C., \& Izumi, L.T. (2004). Putting Education to the Test: A Value-Added Model for California. San Francisco: Pacific Research Institute.

E-MEC (2013). Sistema e-MEC. Recuperado em 16 de janeiro, 2013, de http://emec.mec.gov.br

Eskew, R. K. \& Faley, R H. (1988). Some determinants of student performance in the first college-level financial accounting course. Accounting Review, 63(1), pp. 137-147.

Everson, K. C., Feinauer, E., \& Sudweeks, R. R. (2013). Rethinking Teacher Evaluation: A Conversation about Statistical Inferences and Value-Added Models. Harvard Educational Review, 83(2), pp. 349-370.

Fincher, C. (1985). What is value-added education? Research in Higher Education, 22, pp. 395-398.

Goldstein, H. \& Thomas, S. (1996). Using Examination Results as Indicators of School and College Performance. Journal of the Royal Statistical Society, 159(1), pp. 149-163.

Hanushek, E. A. (1986). The economics of schooling: production and efficiency in public schools' Journal of Economic Literature, 24(3), pp. 1141-1177. 
; Taylor, L. L. (1990). Alternative assessments of the performance of schools: measurement of state variations in achievement. The Journal of Human Resources, 25(2), pp. 179-201.

Instituto Nacional de Estudos e Pesquisas Educacionais Anísio Teixeira. (2011) Enade. Relatório Síntese 2009. Recuperado em 4 de fevereiro, 2013, de http://portal.inep.gov.br/web/guest/relatorio-sintese-2009.

. (2011). Nota Técnica MEC/INEP/DAES No 29/2012. Recuperado em 1 de julho, 2013, de http:// download.inep.gov.br/educacao_superior/enadel

. (2013) Sinaes. Recuperado em 4 de fevereiro, 2013, de http://portal.inep.gov.br/superior-sinaes

(2013b) Perguntas Frequentes. Recuperado em 4 de fevereiro, 2013, de http://portal.inep.gov.br/ perguntas-frequentes 1

Jakubowski, M. (2008). Implementing value-added models of school assessment, EUI RSCAS Working Paper. European University Institute. Recuperado em 4 de fevereiro, 2013, de: http://ideas.repec. $\mathrm{org} / \mathrm{p} / \mathrm{rsc} / \mathrm{rsceui} / 2008-06 . \mathrm{html}$

Keeves, J. P., Hungi, N., \& Afrassa, T. (2005). Measuring value added effect across schools: Should schools be compared in performance? Studies in Educational Evaluation, 31(2), pp. 247-266. doi: 10.1016/j. stueduc.2005.05.012

Kelchen, R. \& Harris, D. N. (2012). Can "Value Added" Methods Improve the Measurement of College Performance? Empirical Analyses and Policy Implications. Context for Success Working Paper.

Kong, W. \& Fu, T. (2012). Assessing the performance of business colleges in Taiwan using data envelopment analysis and student based value-added performance indicators. Omega, 40(5), pp. 541-549. doi: 10.1016/j.omega.2011.10.004

Ladd, H. F. \& Walsh, R.P. (2002). Implementing Value-Added Measures of School Effectiveness: Getting the Incentives Right. Economics of Education Review, 21(1), pp. 1-17. doi: 10.1016/S0272$-7757(00) 00039-\mathrm{X}$

Lareau, A. (1987). Social Class Difference in Family-School Relationships: The Importance of Cultural Capital. Sociology of Education, 60(2), 73-85.

Lei no ${ }^{\circ}$ 10.861, (2004). Institui o Sistema Nacional de Avaliação da Educação Superior - SINAES e dá outras providências. Brasília, DF; Casa Civil - Subchefia de Assuntos Jurídicos.

Malach, J. \& Malcík, M. (2010). Value-added assessment in postsecondary schools, theoretical approaches and research results in the Czech Republic. Kultura i Edukacja, 79(5), pp. 123-153.

McDonnell, S., Chellman, C. C., Littman, C. B., \& Crook, D. (2013). Implementing Value-Added Accountability Measures at the City University of New York. CUNY Office of Policy Research Working Paper.

Meyer, R. H. (1997). Value-Added Indicators of School Performance: A Primer. Economics of Education Review, 16(3), pp. 283-301. doi: 10.1016/S0272-7757(96)00081-7

Meyer, R. H \& Dokumaci, E. (2010). Value-added models and the next generation of assessments. ETS Center for K-12 Assessment and Performance Management. Recuperado em 20 de fevereiro, 2013, de http://www.k12center.org/rsc/pdf/MeyerDokumaciPresenterSession4.pdf.

Miranda, G. J., Casa Nova, S. P. C., \& Cornacchione Júnior, E. B. (2012). Os Saberes dos Professores-Referência no Ensino de Contabilidade. Revista Contabilidade \& Finanças, São Paulo, 23(59), pp. $142-$ 153. doi: 10.1590/S1519-70772012000200006

Papay, J. P. (2012). Refocusing the debate: Assessing the purposes and tools of teacher evaluation. Harvard Educational Review, 82(1), pp.123-141.

Parisotto, I. R. S., Grande, J. F. \& Fernandes, F. C. (2006). O processo ensino e aprendizagem na formação do profissional contábil: uma visão acadêmica. Anais do Congresso USP de iniciação científica em contabilidade, São Paulo, SP, Brasil, 3. 
Raudenbush, S. (2004). What are value-added models estimating and what does this imply for statistical practice? Journal of Educational and Behavioral Statistics, 29(1), pp. 121-129.

Saavedra, A. R. \& Saavedra, J. E. (2011) Do colleges cultivate critical thinking, problem solving, writing and interpersonal skills? Economics of Education Review, 30(6), pp. 1516-1526. doi: 10.1016/j.econedurev.2011.08.006

Santos, N. A. (2012). Determinantes do desempenho acadêmico dos alunos dos cursos de ciências contábeis. Tese de Doutorando em Controladoria e Contabilidade da Faculdade de Economia, Administração e Contabilidade, Universidade de São Paulo, São Paulo, SP, Brasil.

Soares, J. F. \& Ribeiro, L. M.; \& Castro, C. M. (2001). Valor agregado de instituições de ensino superior em Minas Gerais para os cursos de direito, administração e engenharia civil. Dados, 44(2), pp. 363-396.

Souza, E. \& Machado, L. S. (2011). Determinantes do Desempenho dos Cursos de Ciências Contábeis. Anais do Congresso USP de Controladoria e Contabilidade, São Paulo, SP, Brasil, 11.

Taylor, J. \& Nguyen, A. N. (2006). An analysis of the value added by secondary schools in England: is the value added indicator of any value? Oxford Bulletin of Economics and Statistics, 68(2), pp. 203-224.

Wright, D. L., Fox, W. F., Murray, M. N., Carruthers, C. K., \& Thrall, G. (2012). College Participation, Persistence, Graduation and Labor Market Outcomes: An Input-Adjusted Framework for Assessing the Effectiveness of Tennessee's Higher Education Institutions. Context for Success Working Paper Series.

Yunker, J. A. (2005).The dubious utility of the value-added concept in higher education: the case of accounting. Economics of Education Review, 24(3), pp. 355-367. doi: 10.1016/j.econedurev.2004.06.003

Zhang, L. (2009). A value-added estimate of higher education quality of US States. Education Economics, 17(4), pp. 469-489. doi: 10.1080/09645290701838079 